\title{
Prevention and Control of COVID-19 by Primary Health Care Facilities in China: A Field- Survey- Based Qualitative Study in Three Typical Cities
}

\section{Yunyun Yan}

Zhongshan Hospital, Shanghai Medical College of Fudan University

\section{Teng-yang Fan}

Affiliated Hospital of Zunyi Medical University

\section{Yan-ling Zheng}

Wuchang District Shouyilu Street Community Health Care Center

\section{Hai-qin Yang}

Wuchang District Huanghelou Street Community Health Care Center

\section{Tian-shu Li}

Yachuan Township health center, Fenggang County, Guizhou Province

\section{Hai-tang Wang}

Pudong New District Shanggang Community Health Care Center, Shanghai

\section{Yan-feng Gu}

Pudong New District Shanggang Community Health Care Center, Shanghai

\section{Xue Xiao}

Affiliated Hospital of Zunyi Medical University

\section{Zhao-hui Du}

Pudong New District Shanggang Community Health Care Center, Shanghai

Xiao-ming Sun ( $\nabla$ xm_sun2003@163.com )

Zhongshan Hospital, Shanghai Medical College of Fudan University

\section{Research Article}

Keywords: COVID-19, community-based, prevention and control, PHC facilities

Posted Date: June 2nd, 2021

DOI: https://doi.org/10.21203/rs.3.rs-540798/v1

License: (1) This work is licensed under a Creative Commons Attribution 4.0 International License. Read Full License 
Version of Record: A version of this preprint was published at BMC Health Services Research on March 26th, 2022. See the published version at https://doi.org/10.1186/s12913-022-07770-4. 


\section{Abstract}

Background In fulfilling the COVID-19 containment, primary health care (PHC) facilities in China played an important role. We sought to investigate the exact tasks performed at the PHC facilities and the processes of COVID-19 prevention and control.

Methods Semi-structured face-to-face interviews for primary care physicians (PCPs) and a simple survey for residents were conducted in the field survey. Based-on purposive stratified sampling, 32 PCPs were selected from $22 \mathrm{PHC}$ facilities in Wuhan as a high-risk city, in Shanghai as medium-risk city and in Zunyi as low-risk city. In the field survey, semi-structured face-to-face interviews were conducted with PCPs to summarize the tasks of COVID-19 prevention and control at the PHC facilities. A simple survey was used to investigate the local residents' awareness about COVID-19 prevention and control.

Results In pre-outbreak period, the PHC facilities mainly engaged in storing medical supplies; in out-break period, they were responsible for screening, transferring, quarantine and treatment; in regular prevention and control period, attention was given to the employees and items of cold-chain \& fresh food markets, etc. In Wuhan, PHC facilities focused on graded diagnoses and treatments of patients; in Shanghai, they were mainly engaged in at-home/centralized quarantine; in Zunyi, they focused on the screening of highrisk individuals. In urban areas, COVID-19 were more likely to be transmitted; in urban-rural areas, it was difficult to perform screening on the migrant populations; in rural areas, the risk was much lower. The community residents had satisfactory compliance with the preventive measures.

Conclusion We identified differences in the prevention and control tasks performed at the PHC facilities in China. During the different phases of the pandemic, the tasks were adjusted depending on the gradually comprehensive understanding of COVID-19. Among the cities at different risk levels, screening, quarantine, transferring or treatment was chosen to be a priority accordingly. Located in different intra-city geographic locations at different risk levels, the PHC facilities conducted their own tasks accordingly. Additionally, compliance on the part of the local community residents could not be overemphasized in COVID-19 prevention and control.

\section{Background}

In the on-going global pandemic of coronavirus disease 2019, or COVID-19, China has achieved well globally recognized progresses in its prevention and control. As of January 18,2021 , approximately 97.46 million people worldwide had been infected with severe acute respiratory syndrome coronavirus 2 (SARS-CoV-2), which caused more than 2.11 million deaths. ${ }^{1}$ A total of 90,000 COVID-19 cases had been diagnosed in China, and the number of deaths was fewer than $5,000 .{ }^{2}$ As a large country with approximately $18 \%$ of the world's population, China accounts for only $0.1 \%$ of global COVID-19 cases and $0.2 \%$ of deaths. In 2020, the curve of the COVID-19 pandemic in China started to level off, whereas the global trend began to increase substantially and continuously (Fig. 1). 
The PHC facilities played an important role in community COVID-19 prevention and control, growing to better understand the disease and the changing trend of COVID-19 pandemic locally and globally, with their focus shifted accordingly. ${ }^{3}$ We searched PubMed for literatures published in English, China National Knowledge Infrastructure (CNKI) and Wanfang Database for literatures published in Chinese, official website of health commissions at all levels for government policy papers and guidelines, and online academic conference between Jan 1,2020, and Oct 1, 2020, assessing the tasks of primary health care (PHC) facilities in COVID-19 prevention and control during its pandemic in China. However, it was seldom reported about what the $\mathrm{PHC}$ facilities exactly responsible for.

During the COVID-19 pandemic, the preventive and controlling policies were enacted here in China on the principle of region-specific measures, and its cities/areas were classified as high-risk, medium-risk, and low-risk areas. Additionally, COVID-19 pandemic was summarized to have three periods, which referred to the pre-outbreak, outbreak period and regular prevention and control period. ${ }^{4}$ It should be mentioned that the medical resources are unevenly distributed in China. 5,6

To further investigate the exact tasks of the PHC facilities in the prevention and control of COVID-19, we conducted field surveys among the facilities in three typical cities of China: Wuhan as high-risk in Hubei Province, Shanghai as medium-risk and Zunyi as low-risk in Guizhou Province. The chosen cities represented three different levels of economic development regions in China. Shanghai an international economically developed city in eastern China, Wuhan a megacity in central China, and Zunyi an economically underdeveloped city in western China.

\section{Methods}

\section{Study design}

Semi-structured face-to-face interviews were conducted with primary care physicians (PCPs) from the PHC facilities, accompanied by a field survey on the local residents.

The interview items with primary care physicians (PCPs) from the PHC facilities were covered as follows: i) the tasks of the PHC facility with respect to COVID-19 prevention and control; ii) the differences in the prevention and control priorities and tasks during the pre-outbreak, outbreak, and regular prevention and control periods; and iii) the existing problems and suggestions for improvement. The data for PCPs covered age, sex, education, profession, technical title, and working years. The interview took 30-60 minutes.

As to the survey on the local residents, the questions referred to age, sex, education and purpose of hospital visit, attitude toward prevention and control measures taken in the community (5-point Likert scale from strongly supportive to strongly unsupportive), and to the personally taken measures to prevent and contain COVID-19. The survey required 2-5 minutes for each local resident. 


\section{Participants}

The PHC facilities were chosen using purposive stratified sampling as follows: i) from Wuhan, Shanghai and Zunyi respectively according to their representative risk levels; ii) in each city 1-2 PHC facilities (community- or township-based) chosen from its urban, urban-rural and rural areas.

One or several PCPs in charge of COVID-19 prevention and control at each PHC facility were invited to the face-to-face interview, who managerially served as the director or deputy director fully engaged in preventing and containing COVID-19.

In the field survey of the PHC facilities in Shanghai, opportunistic sampling was used to select 10 visiting residents or attendants from each facility for the investigation of COVID-19 prevention and control awareness.

\section{Data collection procedures}

The field survey was conducted from November to December, 2020. Rigor was addressed through indexing the subsets of data across 2 researchers, comparing concepts and themes continuously, and discussing potential themes and disagreements across authors. The research group concluded that theoretical saturation was reached after 32 interviews.

\section{Statistical analysis}

When transcribed, the recordings were analyzed using Microsoft Excel (version 2019) and IBM SPSS Statistics version 22.0 (IBM Corp, Armonk, NY, USA) by two researchers independently. The descriptive summary data were presented with numbers and percentages, and the qualitative data were collated and classified by two researchers. The descriptive summary data were presented with numbers and percentages, and the qualitative data were collated and classified by two researchers.

The transcribed data were analyzed using framework analysis with the procedures following: data familiarization, indexing, theme collating, thematic framework summary, and interpretation. At the PHC facilities, the preventive and containing tasks were summarized depending on different pandemic periods, risk levels, and intra-city geographic locations both in general and in particular, which adequately contained all themes.

\section{Results}

\section{Basic information of the PHC facilities and PCPs}

In the field surveys, we obtained the first-hand information of $22 \mathrm{PHC}$ facilities from the three different cities, 7 in Shanghai, 6 in Zunyi and 9 in Wuhan (including 1 in Ezhou adjacent to Wuhan), and of 32 PCPs 
responsible for different tasks of COVID-19 prevention and control (Table S1). Of 32 PCPs in the interview (Table 1), 15 PCPs came from the PHC facilities in Shanghai, 8 from Zunyi, and other 9 from Wuhan.

Particularly, 2 of 9 PCPs in Wuhan were infected with SARS-CoV-2, one of them critically ill, but they both came to be cured after standard treatment. 


\begin{tabular}{|c|c|}
\hline $\begin{array}{r}T \\
\text { Participant desc }\end{array}$ & $\begin{array}{l}\text { e } 1 \\
\text { ive characteristics }\end{array}$ \\
\hline Characteristics & No. of participants $(\% ; \mathrm{N}=32)$ \\
\hline Age(yr) & $33-67(45.53 \pm 6.54)$ \\
\hline \multicolumn{2}{|l|}{ Mean $\pm S D$} \\
\hline \multicolumn{2}{|l|}{ Sex } \\
\hline Male & $16(50)$ \\
\hline Female & $16(50)$ \\
\hline \multicolumn{2}{|l|}{ Education } \\
\hline Junior college & $4(12.5)$ \\
\hline College & $24(75)$ \\
\hline Graduate school & $4(12.5)$ \\
\hline \multicolumn{2}{|l|}{ Profession } \\
\hline General medicine & $13(40.63)$ \\
\hline Clinical medicine & $8(25)$ \\
\hline Traditional Chinese medicine & $2(6.25)$ \\
\hline Public Health & $2(6.25)$ \\
\hline Management & $1(3.13)$ \\
\hline Nursing & $5(15.63)$ \\
\hline Stomatology & $1(3.13)$ \\
\hline \multicolumn{2}{|l|}{ Technical title } \\
\hline Senior & $3(9.38)$ \\
\hline Associate senior & $14(43.75)$ \\
\hline Intermediate & $12(37.5)$ \\
\hline Junior & $2(6.25)$ \\
\hline Others* & $1(3.13)$ \\
\hline Years of work experience & $2 \sim 45(21.59 \pm 9.08)$ \\
\hline Mean $\pm S D$ & \\
\hline
\end{tabular}




\section{Task summary of the PHC facilities}

\section{Brief summary}

As shown in Table 2, we made a brief summary of the main tasks of COVID-19 prevention and control at the PHC facilities. In controlling infection sources, the PHC facilities were mainly responsible for screening, transferring, quarantine and treatment. The screening, which focused on high-risk populations who were confirmed/suspected patients, febrile patients, close contacts, and those who returned from the high-risk areas in the country, was performed at homes, travel centers /terminals, PHC facilities (for preexamination and triage), and fever consultation rooms/clinics. Those who were screened out were transferred to at-home/centralized quarantine. Cutting off the transmission route was mainly achieved by disinfection and temporary storage and disposal of waste. The susceptible populations were highly protected, thanks to health education, protective measures, psychological interventions, and COVID-19 vaccines. 


\section{Table 2}

The main tasks of COVID-19 prevention and control by the PHC facilities

\section{Summary Content}

$\begin{array}{lll}\begin{array}{l}\text { Controlling } \\ \text { infection } \\ \text { sources }\end{array} & \begin{array}{l}\text { Four } \\ \text { aspects }\end{array} & \text { Screening, transferring, quarantine, treatment } \\ & & \end{array}$

$\begin{array}{lll}\begin{array}{l}\text { High-risk } \\ \text { populations }\end{array} & \begin{array}{l}\text { Six } \\ \text { groups }\end{array} & \begin{array}{l}\text { Confirmed patients, patients suspected of infection, close contacts, } \\ \text { febrile patients, individuals from high-risk areas, employees of cold- } \\ \text { chain/fresh markets and other persons concerned }\end{array}\end{array}$

Screening Four Screening at travel centers/intervals, house-by-house screening, preforms examination/triage, fever consultation rooms/clinics, NAT on high-risk individuals

Quarantine Two At-home/centralized quarantine and observation forms

Cutting off Two Disinfection, temporary storage and disposal of waste transmission ways routes
Protecting
Four susceptible ways Protective measures (face mask, hand washing, ventilation, social distancing), populations

Health education, psychological interventions, vaccination

PHC = primary health care, NAT= nucleic acid testing

\section{Flowchart of the main tasks}

As indicated in Figure 2, the main tasks of COVID-19 prevention and control at the PHC facilities were to assist the Center for Disease Control and Prevention (CDC) in contact tracing and epidemiological investigation, and to screen high-risk populations at the travel centers/intervals, at the community households, as well as within the PHC facilities. Those who had returned from medium- and high-risk areas and their close contacts were transferred to at-home/centralized quarantine, and those who had fever to a fever consultation room or a fever clinic. Nucleic acid testing (NAT) was performed on the highrisk populations and febrile patients, and those who were tested positive were transferred to the designated hospital for a standard treatment.

\section{Perspective 1: Tasks of the PHC facilities during different pandemic phrases}




\section{During the pre-outbreak period (Table S2), inadequate understanding and a shortage of medical supplies was the main problem.}

The understanding of COVID-19 and SARS-CoV-2 was limited during the pre-outbreak period. There was a severe shortage of medical supplies at the PHC facilities to mitigate the respiratory infectious disease of COVID-19 even though the raising and donating were active across the world.

\section{During the outbreak period, infections' treatment, closed- loop management of the febrile patients, centralized quarantine and psychological intervention were all important work at the PHC facilities.}

During the field investigation, 6 of 9 PHC facilities in Wuhan took the responsibility of treating the infected patients, which was significantly different than in other cities. Quite a number of general wards were temporarily converted to quarantine wards to accommodate a growing demand for sick beds (Figure S1). Since fever was a typical and initial symptom of COVID-19 infections, febrile patients were high-risk individuals to be screened out for closed-loop management (Figure S2). Initially, at-home quarantine was recommended (Figure S3), but the problem was that cross-infection could not be strictly avoided as this respiratory infectious disease was transmitted rapidly through droplets or daily contacts, so that centralized quarantine became the main management style (Figure S4). During the pandemic of COVID19 , infected patients, suspected patients, people under quarantine, medical workers as well as populations in general were very likely to suffer a mental pressure, in case of which, psychological intervention became extremely important.

\section{In the regular prevention and control period (Table S3), NAT on cold-chain/fresh foods and their logistics and populations were priorities at PHC facilities.}

The PHC facilities began to perform several newly required tasks during the regular period of prevention and control, which referred to the sample collection of SARS-CoV-2 NAT results from employees and coldchain/fresh foods and their logistics for the purpose of containing infection sources. Regular NAT was also conducted in such high-risk populations as medical workers who had a potentially occupational 
exposure risk to SARS-CoV-2, and the staff of nursing homes, since the seniors were more vulnerable to SARS-CoV-2.

\section{Perspective 2: Tasks of the PHC facilities at different risk levels (Table S4)}

\section{In the high-risk city of Wuhan, graded diagnoses and treatments and timely transferring were major tasks.}

Wuhan, a megacity in central China with a population of about 11 million, was nationally reported to have about 50,000 confirmed COVID-19 cases as of Mar 15, 2021. At the PHC facilities, where graded diagnoses and treatments were performed on the patients with different symptoms, managed the confirmed patients with mild symptoms and suspected patients, and transferred the critically ill patients after diagnosis. As a growing number of high-risk individuals needed to be transferred, ambulances were not enough; thus they were only used to meet the need of critically ill patients for transferring.

\section{In the medium-risk city of Shanghai, returnees from high- areas abroad were managed by rigid at-home/centralized quarantine. Sentinel clinics for fever patients were also established at PHC facilities.}

Shanghai, an internationally metropolitan city in eastern China, has a population of about 24 million, about 371 local cases and 1,400 imported cases were identified as of Mar 15, 2021. Those who had been screened out to be high-risk individuals were supposed to be transferred by ambulance. With the largest mobile population and most visitor arrivals in the country, the city of Shanghai had its PHC facilities responsible for returnees' management from abroad. The individuals who were screened out at the airport (Figure S5) were required to follow quarantine. At-home quarantine places a high demand on the living conditions, on the ground that the high population density in the city could increase the possibility of family cluster cases of COVID-19 infection.

\section{In the low-risk city of Zunyi, febrile patient management and screening were top priorities.}

Zunyi, a city in western China, has a population of about 6 million. During the outbreak period of COVID-19 pandemic, a number of 32 individuals were tested positive. When screened out, the febrile patients had to be transferred to the fever clinic of the designed secondary hospital. As a low-risk area, Zunyi had its tasks focus on screening and management of high-risk individuals (Figure S6). 


\section{Perspective 3: Tasks of the PHC facilities at different intra- city locations}

In China, the urban areas, which tend to have a high population density, are more likely to cause the spread of infectious diseases like COVID-19. In the urban-rural areas, the migrant populations were difficult to screen in the urban-rural areas during the pandemic of COVID-19. In the rural areas, which have much to be desired in economics and infrastructure, tend to have limited access to medical resources. Accordingly, the rural citizens were at a relatively lower risk with infectious disease which could be transmitted by close contact with infected patients.

\section{Local residents' awareness of COVID-19 prevention and control}

In the survey of personally taken measures for prevention and control on the part of the community residents (Table 3), such as face mask wearing, frequent hand washing, proper disinfection, prompt ventilation, health code adherence, physical exercise and mood management, $76.27 \%$ of them reported their taking five measures or more. During the outbreak period, $86.44 \%$ of the residents reported their complete support for the community-based prevention and control measures such as lockdown to avoid public gatherings, surface disinfection in the public places, and health education in the form of bulletin boards. 


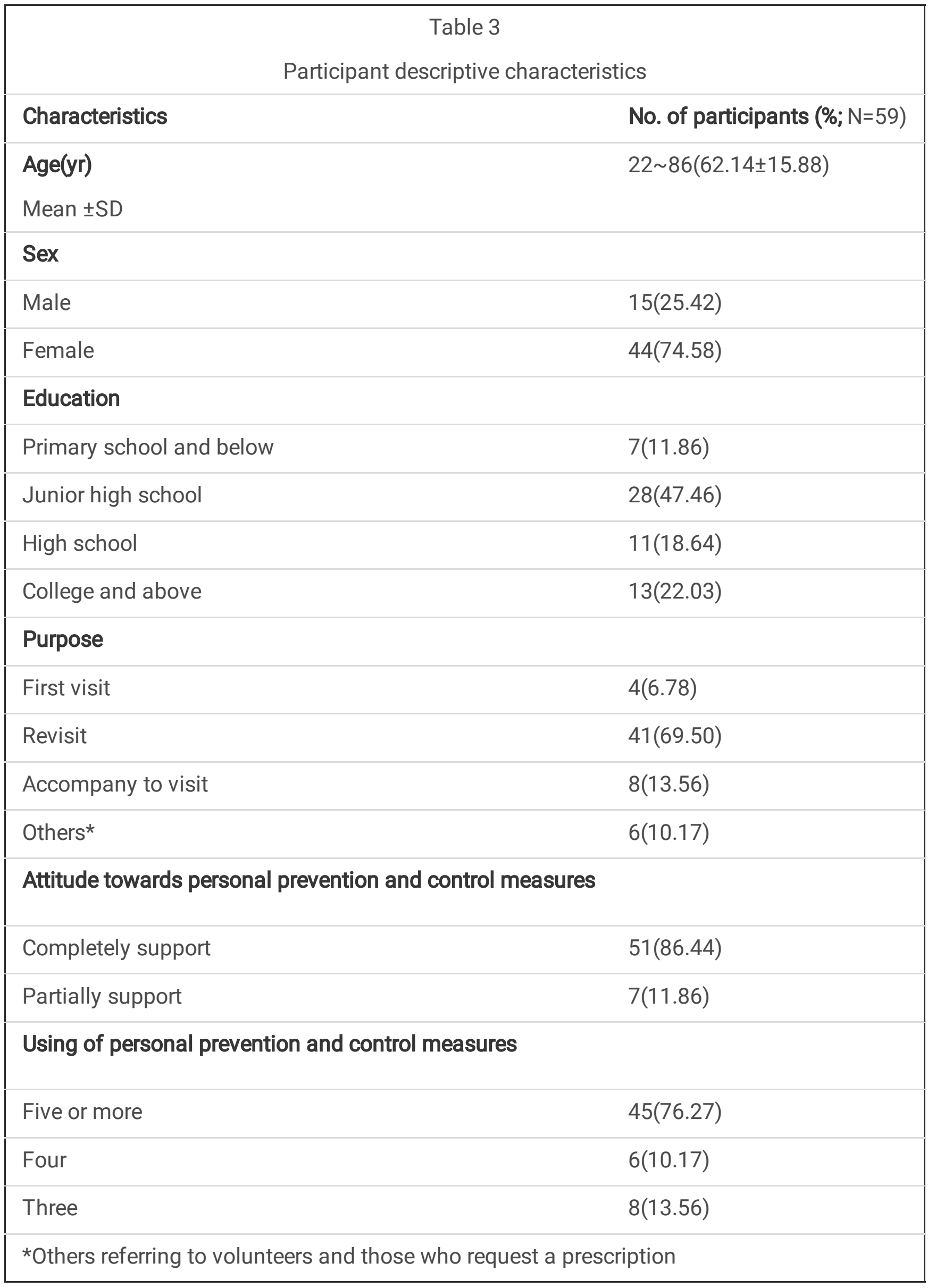




\section{Discussion}

\section{Statement of principal findings}

To our knowledge, this was the first study on the community-based preventive and controlling measures against COVID-19 manipulated by the PHC facilities in China. We identified differences in the tasks of the PHC facilities depending on different periods of the pandemic, in the cities at different risk levels, and in the intra-city geographic locations. During the community-based mitigation of COVID-19, the PHC facilities were critically needed.

\section{Strengths and limitations}

This is a field-survey-based study of the tasks performed at PHC facilities in China during the COVID-19 pandemic, and face-to-face interviews were conducted with PCPs from different intra-city geographic locations in three typical cities including Wuhan, Shanghai, and Zunyi. The tasks at PHC facilities in different periods of the pandemic were investigated. But there are several limitations in this research. It did not follow strict random sampling and the sample size of the community-based residents survey was small, and this article did not involve any quantitative data.

\section{Interpretation within the context of the wider literature}

The COVID-19 prevention and control require the involvement of multiple facilities and participation of populations in all aspects. ${ }^{7}$ The implementation of preventive and controlling measures requires the cooperation of residents. ${ }^{8}$ The survey indicated that the residents showed a high level of support and adherence to the regulations and measures of COVID-19 prevention and control, which significantly enhanced the effectiveness of fulfillment. The implementation of some measure may be considered a violation of human rights; therefore, the government should carefully weigh the pros and cons in making a particular decision or issue a particular policy for action. ${ }^{9}$

The survey showed that management of febrile patients was an important strategy for COVID-19 mitigation. To respond to infectious diseases, in China, fever clinics have been established in the secondary hospitals and above, since the outbreak of SARS in 2003. ${ }^{10,11}$ Symptom monitoring of fever plays a key role in the control of infectious diseases. ${ }^{12}$ However, the treatment provided at the fever clinic within the designated hospitals could increase the cost, the risk of cross infection, and the waste of medical resources. During the first half of 2020, pilot fever sentinel clinics in the PHC facilities were established. ${ }^{13}$ The ability to respond to a public health emergency in China are geographically unbalanced, ${ }^{5,6}$ as illustrated in Zunyi of Guizhou province.

Quarantine strategies are important to controlling infectious diseases. ${ }^{14}$ In the pre-outbreak period in China, the high-risk populations were all monitored under at-home quarantine. However, at-home 
quarantine places a high demand on the living conditions, and the high population density in the urban areas could increase the possibility of family cluster cases of COVID-19 infection. ${ }^{15}$ Therefore, centralized quarantine was adopted in Wuhan In Shanghai, and Zunyi during later periods. We recommend the centralized quarantine as the primary form of managing the populations at risk for COVID-19 infection. This recommendation can be actualized by the PHC facilities.Psychological problems resulting from quarantine should not be ignored during the COVID-19 mitigation. ${ }^{16}$ At-home quarantine was likely to increase patient comfort and improve their well-being, ${ }^{17,18}$ whereas centralized quarantine could probably cause psychological problems, such as anxiety and mania. ${ }^{19}$ Therefore, the psychological conditions of individuals should be addressed.

During the outbreak period in China, the cities were divided at the province level of low-, medium-, and high-risk, and the counties were treated as the minimal units to define risk areas for class-specific and region-specific prevention and control. ${ }^{20}$ During the regular prevention and control period, the pandemic was characterized by cluster cases; therefore, the policies were modified to redefine the minimal unit of risk areas. ${ }^{21}$ In the high-risk areas, proper and prompt NAT was conducted on all residents. ${ }^{22}$ With improved understanding of COVID-19, the regular sample collection of SARS-CoV-2 NAT results from the businessmen of cold-chain/fresh food markets, etc. would better help contain the sources of COVID-19 infection.

\section{Implications for policy, practice and research}

Widespread vaccination to achieve herd immunity is the goal of current COVID-19 preventive and controlling efforts. ${ }^{23,24}$ In China, the priority groups for vaccination referred to the medical staffs, nursing home staffs. ${ }^{25}$ Of course, those who did not have any contraindications were allowed to be vaccinated, which was carried out in a stepwise manner. However, the emergence of the so-called British and South African variants ${ }^{26,27}$ has posed a great challenge to the immunity under vaccination. Andreano et al. revealed possible immune escape by these SARS-CoV-2 variants, ${ }^{28}$ which requires maintenance of preventive and controlling measures while focusing on improving vaccine efficacy.

\section{Conclusion}

In our investigation we identified six key specific factors of the PHC facilities in the community-based prevention and control of COVID-19, which referred to screening, febrile patients' management, transferring, quarantine, region-, class- and population-specific accurate management and treatment for COVID-19 patients when necessary. These factors were badly taken into count while mitigating COVID-19 transmission, as there were no specific therapeutic drugs and no vaccines widely available.

\section{Declarations}

\section{Ethics approval and consent to participate}


All methods of this survey were carried out in accordance with relevant guidelines and regulations.

Ethics approval by the Ethics Committee of Pudong Institute for Health Development, Shanghai (PDWFY2020001) was acquired prior to the current study which did not violate any ethical rule. No patient was involved in this research. Oral informed consent was obtained from all participants before the interview, and this procedure was approved by the Ethics Committee of Pudong Institute for Health Development, Shanghai.

\section{Consent for publication}

Not applicable.

\section{Availability of data and materials}

All data relevant to the study was available from the corresponding author on reasonable request.

\section{Competing interests}

All authors declare no interest.

\section{Funding}

Pudong New District Health System Important Weak Discipline (PWZbr2017-07), Pudong New District Health System Preponderant Discipline of General Practice (PWYq2020-01), and Pudong New District Health System New Pilot Model of General Practice Service in Traditional Chinese Medicine (PDZY-20200703).

\section{Authors' Contributions}

Dr. Yan conducted the field surveys included PCPs interviews and resident surveys. Dr. Fan, and Zheng transcribed the audio-recorded materials. Dr. Yang, Li, Wang, and Gu analyzed and interpreted the data. Dr. Yan was the main contributor to writing the manuscript. Dr. Du, Prof. Sun, and Dr. Xiao contacted the PHC facilities and reviewed the manuscript. Dr. Yan, Du, and Prof. Sun designed the research, supervising the entire study process. All authors reviewed and approved the final manuscript.

\section{Acknowledgments}

We thank all the experts who participated in the interviews. We are grateful to our colleagues from Shanghai Pudong New District Shanggang Community Health Care Center, Zhongshan Hospital Fudan 
University, and Affiliated Hospital of Zunyi Medical University and all PHC facilities surveyed for their assistance.

\section{References}

1. World health organization (WHO) Coronavirus Diease (COVID-19) Dashboard.Update on the epidemic of Covid-19, as of 24:00 on January 18, 2021. https://covid19.who.int/, accessed July 18, 2021.

2. National Health Commission of the People's Republic of China.Update on the epidemic of Covid-19, as of $24: 00$ on January 18, 2021. http://www.nhc.gov.cn/xcs/yqtb/list_gzbd.shtml, accessed July 18, 2021.

3. Zhang X, Yang S. "A community system": A critical foundation for the epidemic prevention and control of SARS-CoV-2. The International journal of health planning and management 2020 Sep;35(5):12461249.

4. Hu S L. New normal and new future: summing up and reflection for the future scientific prevention and control COVID-19 to ensure its enduring and steady progress. Fudan University Journal of Medical Sciences 2020;47: 467-71.

5. Gai RY, Zhou CC, Xu LZ, et al. Health resource allocation and productive efficiency of Chinese county hospitals: data from 1993 to 2005. Biosci Trends 2010; 4(5):218-24.

6. Dong $E$, Liu $S$, Chen $M$, et al. Differences in regional distribution and inequality in health-resource allocation at hospital and primary health centre levels: a longitudinal study in Shanghai, China. BMJ Open 2020; 10(7):e035635.

7. Jun Zhang, Yu Liu. Effectiveness of service and reflection on "volunteer + community social organization" in the prevention and control of COVID-19 epidemics. Journal of Tianjin Administration Institute 2020; 22(03):79-86.

8. Galasso V, Pons V, Profeta P, Becher M, Brouard S, Foucault M. Gender differences in COVID-19 attitudes and behavior: Panel evidence from eight countries. Proc Natl Acad Sci U S A 2020; 117(44):27285-27291.

9. Botes WM, Thaldar DW. COVID-19 and quarantine orders: A practical approach. South African medical journal = Suid-Afrikaanse tydskrif vir geneeskunde 2020; 110(6):469-472.

10. Ministry of Health of the People's Republic of China. Notice of the Ministry of Health of China on the Construction of an Infectious Disease Department in Secondary and Above General Hospitals.http://www.nhc.gov.cn/yzygj/s3577/200804/978c829fa9514b95bcba744df293fdee.shtml, accessed July 20, 2021.

11. Ministry of Hygiene, Peoples' Republic of China. Guidelines for Establishment of Fever Outpatient (Emergency) Clinics in Health Care Facilities (Trial Version). Chinese Journal for Clinicians 2003; (06):16-17.

12. Parrella A, Dalton CB, Pearce R, Litt JC, Stocks N. ASPREN surveillance system for influenza-like illness - A comparison with FluTracking and the National Notifiable Diseases Surveillance System. Aust Fam Physician 2009; 38(11):932-6. 


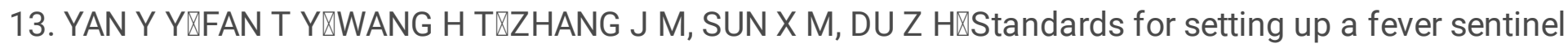
surveillance clinic in primary healthcare institutions $\bigotimes$ findings of a qualitative Delphi study $₫$ Chinese General Practice $₫ 2021,24$ (10) $₫ 1190-1197 \rrbracket$

14. Cui Q, Hu Z, Li Y, Han J, Teng Z, Qian J. Dynamic variations of the COVID-19 disease at different quarantine strategies in Wuhan and mainland China. Journal of infection and public health 2020; 13(6):849-55.

15. Zhu Y, Wang C, Dong L, Xiao M. Home quarantine or centralized quarantine, which is more conducive to fighting COVID-19 pandemic?. Brain, behavior, and immunity 2020, 87:142-43.

16. Brooks SK, Webster RK, Smith LE, et al. The psychological impact of quarantine and how to reduce it: rapid review of the evidence. Lancet 2020; 395:912-920.

17. Lu H, Nie P, Qian L. Do Quarantine Experiences and Attitudes Towards COVID-19 Affect the Distribution of Mental Health in China? A Quantile Regression Analysis. Applied research in quality of life 2020;Jun 29;1-18.

18. Shaban RZ, Nahidi S, Sotomayor-Castillo C, et al. SARS-CoV-2 infection and COVID-19: The lived experience and perceptions of patients in isolation and care in an Australian healthcare setting. $A m J$ Infect Control 2020 Dec;48(12):1445-1450.

19. Gan Y, Ma J, Wu J, Chen Y, Zhu H, Hall BJ. Immediate and delayed psychological effects of provincewide lockdown and personal quarantine during the COVID-19 outbreak in China. Psychological medicine 2020;Aug 13:1-12.

20. Bareau of Medical administration and Hospital Authority, National Health Commission of China. Notice of the General Office of the National Health Commission of China on Further Implementation of Scientific, Accurate, Region-Specific, and Class-Specific Prevention and Treatment Measures for the Management of Medical Services During the Epidemic. http://www.gov.cn/zhengce/zhengceku/202002/27/content_5483867.htm, accessed July 20, 2021.

21. Joint Prevention and Control Mechanism of the State Council. Prevention and Control Protocol for COVID-19 (Trial Version 7). http://www.nhc.gov.cn/jkj/s3577/202009/318683cbfaee4191a ee29cd774b19d8d.shtml, accessed July 20, 2021.

22. Wuhan carried out centralized nucleic acid testing for all residents in the city. China Medical News 2020; 35(10):7.

23. Frederiksen LSF, Zhang Y, Foged C, Thakur A. The Long Road Toward COVID-19 Herd Immunity: Vaccine Platform Technologies and Mass Immunization Strategies. Frontiers in immunology 2020;Jul 21;11:1817.

24. Calina D, Docea AO, Petrakis D, et al. Towards effective COVID-19 vaccines: Updates, perspectives and challenges (Review). International journal of molecular medicine 2020; 46(1):3-16.

25. Chinese Center for Disease Control and Prevention. Technical Guidelines for Influenza Vaccination in China (2020-2021). http://wwwchinacdccn/jkzt/crb/bl/lxxgm/jszl_2251/202009/t2020091 0_219046html, accessed July 20, 2021. 
26. Bin Arif T. 501.V2 and B.1.1.7 variants of COVID-19: a new time-bomb in the making?. Infect Control Hosp Epidemio/ 2021;Jan 11;1-2.

27. Leung K, Shum MH, Leung GM, Lam TT, Wu JT. Early transmissibility assessment of the N501Y mutant strains of SARS-CoV-2 in the United Kingdom, October to November 2020. Euro Surveill 2021 Jan;26(1):2002106.

28. Andreano E, Piccini G, Licastro D, et al. SARS-CoV-2 escape in vitro from a highly neutralizing COVID19 convalescent plasma. bioRxiv : the preprint server for biology 2020;Dec 28.424451.

\section{Figures}
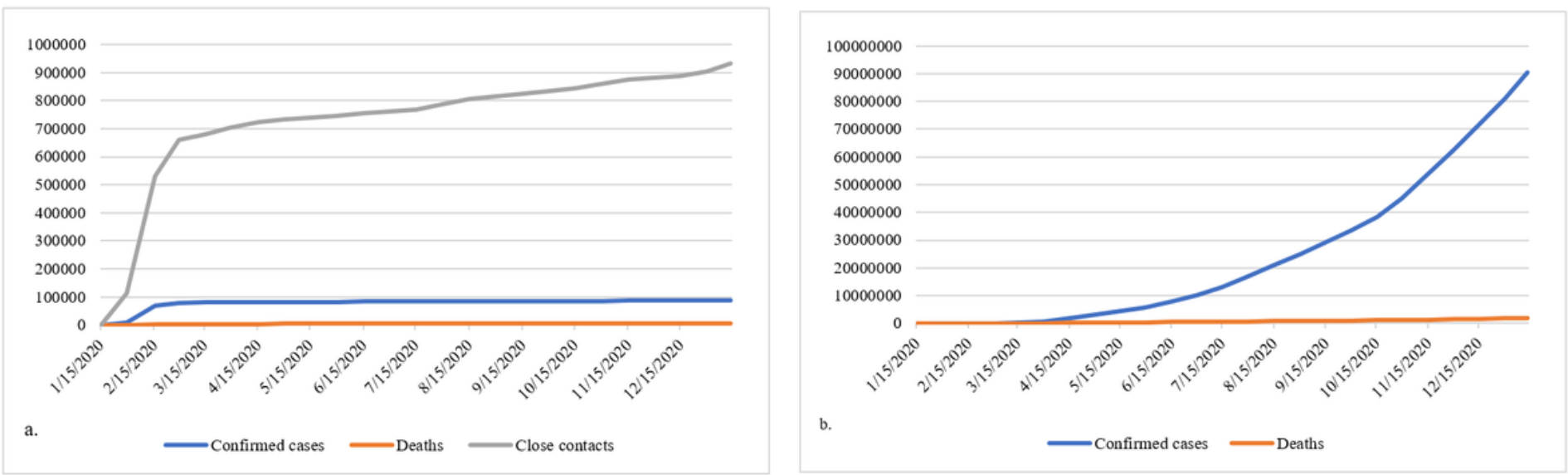

\section{Figure 1}

Trend of COVID-19 pandemic in China (a) and in the world (b) The data of the pandemic in China were derived from the official website of National Health Commission, and the global data from the official website of World Health Organization (WHO) 


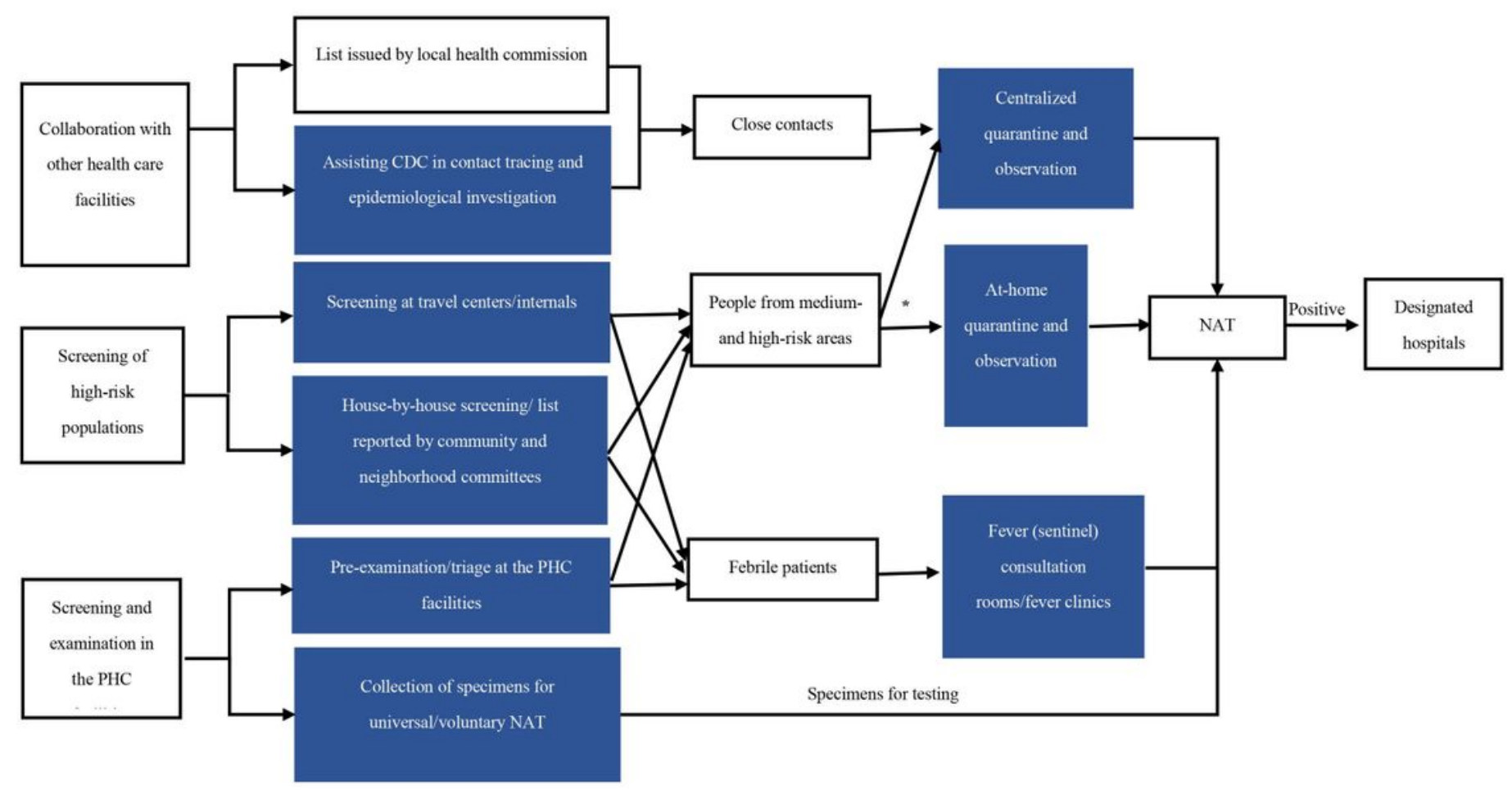

Figure 2

Flowchart of COVID-19 prevention and control tasks by the PHC facilities CDC=Center for Disease Control and Prevention, PHC = primary health care, NAT=nucleic acid testing. Nodes in blue as the main tasks in the PHC facilities. * Special populations refer to older people (age 70 years and above), minors (age 14 years and below), women who are pregnant or in traditional postpartum confinement, people with mobility difficulties, people with older people or children in need of care, and people with underlying diseases and are not suitable for centralized quarantine. Special populations should be diagnosed using nucleic acid testing at examination sites. Those with negative results and proper housing conditions can apply for athome quarantine and health observation after strict evaluation and approval

\section{Supplementary Files}

This is a list of supplementary files associated with this preprint. Click to download.

- SupplementaryMaterials0520.docx 